Research Article

\title{
Ground Simulation Test of 2D Dynamic Overload Environment of Fuze Launching
}

\author{
Zhibo Wu $\left(\mathbb{D}\right.$, Tiehua Ma, Yanbing Zhang, and Hongyan Zhang ${ }^{1}$ \\ Science and Technology on Electronic Test and Measurement Laboratory, North University of China, Taiyuan 030051, China \\ Correspondence should be addressed to Zhibo Wu; b1507006@st.nuc.edu.cn
}

Received 31 August 2019; Revised 16 December 2019; Accepted 30 January 2020; Published 19 February 2020

Academic Editor: Chengzhi Shi

Copyright (C) 2020 Zhibo Wu et al. This is an open access article distributed under the Creative Commons Attribution License, which permits unrestricted use, distribution, and reproduction in any medium, provided the original work is properly cited.

\begin{abstract}
The fuze launch process is subjected to backseat and spin overloads. To address this issue, a loading method of a 2D dynamic acceleration environment was developed in this study for testing fuze antioverload performance on ground. The techniques of flywheel energy storage, high-speed impact, and centrifugal rotation in the track are combined in a dynamic analysis and simulation. First, the flywheel is rotated at a constant speed by a variable-frequency motor to obtain high kinetic energy. Second, an impact hammer is instantaneously released on the specimen at a high speed, loading the backseat acceleration environment. Finally, the impact hammer is retracted, and the specimen is rotated in the track instead of spinning around its axis, thereby loading the centrifugal acceleration environment. The peak value and pulse width of the $2 \mathrm{D}$ overload acceleration can be adjusted by changing the speed of the flywheel and buffers in the abovementioned process. The experimental and simulation results observed that the peak value of backseat acceleration could reach $34,559 \mathrm{~g}$, the pulse width was approximately $400 \mu \mathrm{s}$, and the peak value of the centrifugal acceleration was $1,020 \mathrm{~g}$. The study results showed that the proposed approach fulfills the requirements of the $2 \mathrm{D}$ overload simulation test of the micro-electromechanical system (MEMS) fuze safety and arming mechanism. The proposed loading method has been successfully applied to ground simulation tests of the MEMS fuze safety and arming mechanism.
\end{abstract}

\section{Introduction}

The fuze is an important component of ammunition and the final execution device of a weapon system. It plays a defining role in determining the success or failure of weapons deployed against a target [1]. With the rapid development of fuze technology, new challenges have emerged in terms of fuze design and assessment. Consequently, fuze-launching ground simulation technology has become increasingly important.

Traditional fuze simulation technology includes impact loading and centrifugal loading methods. The former mainly includes the vertical drop weight [2-4], Hopkinson bar $[2,5]$, Taylor bar $[2,6]$, gas gun $[7,8]$, and electromagnetic gun techniques $[9,10]$, while the latter includes the improved centrifuge [11] and rotary testing machine. However, the abovementioned approaches have limitations in that they separate backseat and centrifugal overloads and are thus unable to simulate the actual fuze launch environment. Therefore, it is critical to conduct research on fuze ground simulation technology in $2 \mathrm{D}$ dynamic acceleration environments.

The aim of fuze simulation technology in 2D dynamic acceleration environments is to simulate the influence of backseat and spin overloads in the gun tube during firing. Xiang et al. [12] designed a centrifuge with a radial rotating mechanism. The centrifuge rotation can simulate the effect of backseat overload on a fuze. A motor is installed in the radial rotating mechanism to rotate the fuze and simulate the effect of fuze centrifugal overload. Wang et al. [13] used a motor and torsion spring to drive the rotating plate and specimen, respectively, which decomposes centrifugal acceleration. The peak value and pulse width of $2 \mathrm{D}$ acceleration can be adjusted by changing the speed of the motor and the torque of the torsion spring within a certain range.

Zhang et al. [14] used an air gun to drive a centrifuge capable of starting instantly. The angle displacement of the specimen is controlled by a single-axis vector turntable. The 
resultant centrifuge acceleration decomposes into axial and transverse components and further simulates missile-launch dual-environmental forces. However, these methods have the limitations of a complex system structure, a smaller simulated overload peak, and difficulty in performing high overload simulations of a large-diameter projectile fuze.

Meanwhile, Qi et al. [15] and Yang et al. [16] proposed a method of removing fuze insurance based on the gas gun impact and rotating tube receipt. The pulse width of the overload acceleration is effectively prolonged by using foam aluminum as a buffer. Qian et al. [17] and Xu et al. [18] simulated and tested the performance of a fuze liquid storage battery using a shock rotation method. Lin [19] studied simulation technology of a fuze-launching backseat and rotating dual environment based on electromagnetic emission. Although these methods cover a wide application area, they involve significant costs and complex operations, which are not conducive to the future development of fuze simulation technology.

In this paper, a loading method of fuze $2 \mathrm{D}$ dynamic acceleration environments is therefore proposed. The techniques of flywheel energy storage, high-speed impact, and centrifugal rotation in the track are combined based on dynamic analysis and a simulation. The designed loading system of a $2 \mathrm{D}$ dynamic acceleration environment is presented. The flywheel is driven to rotate at a set value by a variable-frequency motor in the system. The impact hammer is instantly released, striking the specimen at a high speed and loading the backseat acceleration environment. Thereafter, the impact hammer is retracted. The specimen rotates in the track instead of rotating around its axis, thereby loading the centrifugal acceleration environment. The flywheel speed can be changed and various buffers can be used in the abovementioned process to adjust the peak value and pulse width of the $2 \mathrm{D}$ overload acceleration.

The remainder of this paper is organized as follows. The structure and working principle of the loading system are introduced in Section 2. The acceleration-environment loading system is described in Section 3 based on a dynamic analysis. In Section 4, the effectiveness of the fuzzy proportional integral derivative (PID) mode, which is adopted to adjust the speed of the variable-frequency motor, is validated by the simulation results. The simulation process of backseat and centrifugal overload environments is described in Section 5 with the simulation test results. The conclusions of this study are presented in Section 6.

\section{Structure and Principle of the Loading System}

The loading system must accommodate the continuous loading of $2 \mathrm{D}$ dynamic overload environments with backseat acceleration up to $20,000 \mathrm{~g}$, a pulse width up to $300 \mu \mathrm{s}$, and centrifugal acceleration up to $1,000 \mathrm{~g}$. The loading system structure is composed of a flywheel energy storage subsystem, a 2D dynamic overload environment loading device, and a test specimen, as shown in Figure 1. The flywheel energy storage subsystem mainly includes a variable-frequency motor and a clutch, spindle, and flywheel. The subsystem is the main hardware of the loading system, which provides kinetic energy for the simulation test. The flywheel radius is $0.5 \mathrm{~m}$. Driven by the variable-frequency motor, the flywheel can achieve a high speed.

The 2D dynamic overload environment loading device primarily includes a centrifugal track, impact hammer, limit groove, cylinder, brake disc, and brakes. The centrifugal track radius is $0.6 \mathrm{~m}$. The impact hammer is installed in the limit groove and can move along it. A wire rope is connected to the impact hammer and wound around the cylinder. The release and retract of the hammer are controlled by the two brakes, which enables the $2 \mathrm{D}$ dynamic overload environment to be realized.

The test specimen primarily includes the micro-electromechanical system (MEMS) fuze safety and arming mechanism, two acceleration sensors, and a memory test circuit. The test specimen is initially placed in the centrifugal track. The two acceleration sensors, respectively, point to the tangential and radial directions of the centrifugal track. They can record the results of the simulation test process. In addition, the sensors provide the basis for evaluating the working condition of the $2 \mathrm{D}$ dynamic environment loading system and the performance of the MEMS fuze safety and arming mechanism.

The working process of the loading system is divided into the flywheel energy storage, backseat-overload loading, and centrifugal-overload loading, as shown in Figure 2.

2.1. Flywheel Energy Storage. At the beginning of the test, the specimen with the MEMS fuze safety and arming mechanism and the $2 \mathrm{D}$ acceleration storage test device is stationary in the centrifugal track. Brake A latches the brake disc and brake B loosens it. Then, the clutch is engaged, the variablefrequency motor is started, and the flywheel is driven to rotate at the set speed. The impact hammer rotates with the flywheel. As the brake disc is latched, the wire rope is wound around the cylinder. Under the constraint of the limit groove, the impact hammer is retracted back to the bottom of the limit groove by the wire rope. Hence, the flywheel, cylinder, and impact hammer, which are relatively static, rotate together.

2.2. Backseat-Overload Loading. When the flywheel reaches the set speed and stabilizes, the position sensor detects that the flywheel is rotating to the specified position, and the clutch disconnects. This function prevents damage to the motor caused by an immense impact. Brake A loosens, and the impact hammer is thrown outward along the limit groove under the action of centrifugal force, and it extends into the centrifugal track. The impact hammer collides with the specimen at high speed to load the backseat-overload environment.

2.3. Centrifugal-Overload Loading. After the collision, the specimen gains a certain velocity and rotates along the centrifugal track. Owing to centrifugal force, the specimen is loaded with centrifugal overload. It should be noted that, since the mass of the specimen is very small, the specimen 


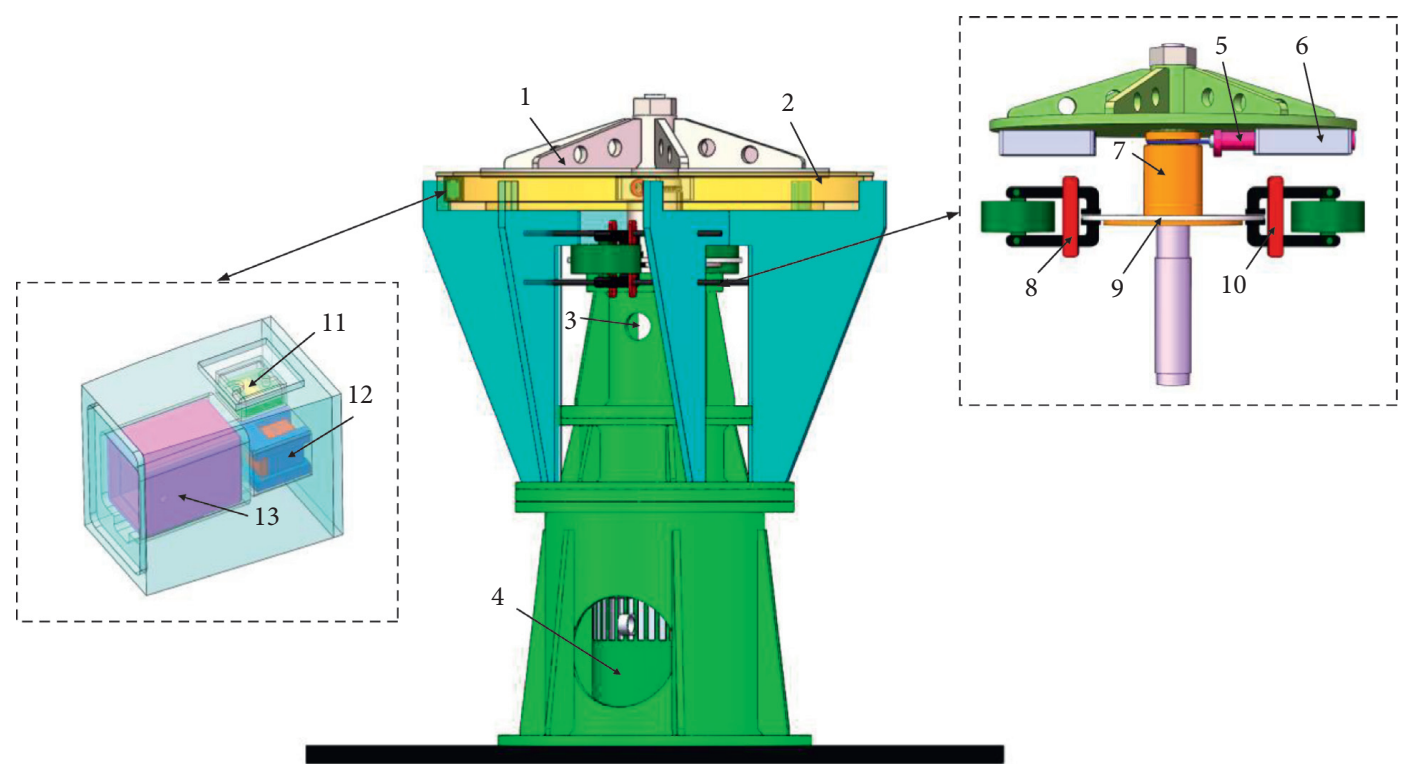

Figure 1: Structure of the loading system of the 2D dynamic overload environment. (1) Flywheel. (2) Centrifugal track. (3) Spindle. (4) Frequency-variable motor. (5) Impact hammer. (6) Limit groove. (7) Cylinder. (8) Electromagnetic brake A (9) Brake disc. (10) Electromagnetic brake B (11) MEMS fuze safety and arming mechanism. (12) 2D acceleration sensors. (13) Memory test circuit.

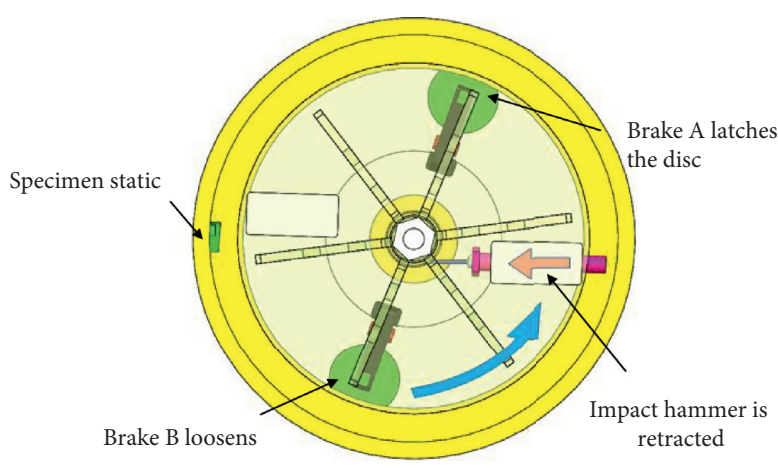

(a)

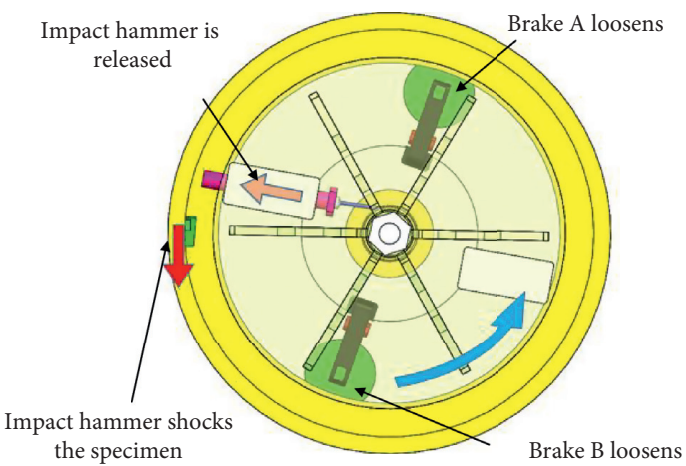

(b)

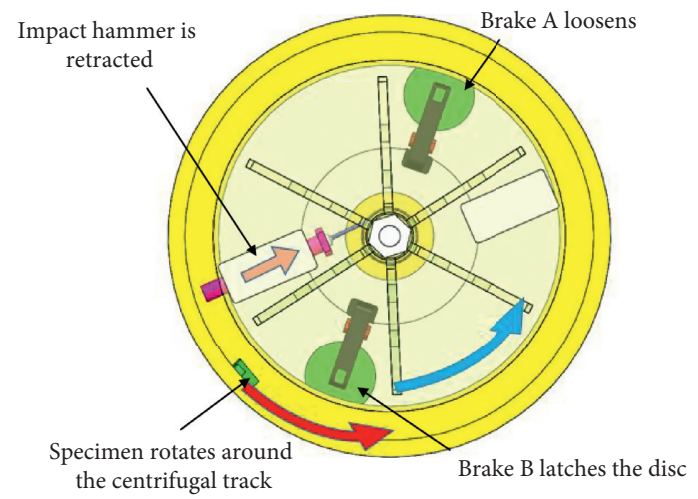

(c)

FIGURE 2: Working principle of the loading system of the 2D dynamic acceleration environment. (a) Flywheel energy storage. (b) Backseatoverload loading. (c) Centrifugal-overload loading.

velocity after collision is greater than that of the impact hammer; thus, it will have a secondary collision with the impact hammer. To avoid this phenomenon, brake B is used in the loading system. After the impact hammer collides with the specimen, the brake disc is latched by brake B, the steel wire rope is wound around the cylinder, and the impact hammer is then retracted back to the bottom of the limit groove. 
The loading system control block diagram, including the control loop of the flywheel energy storage subsystem and the $2 \mathrm{D}$ acceleration loading control loop, is shown in Figure 3. The loading control process is divided into four steps. (1) The industrial personal computer (IPC) controls the clutch engagement, brake A latching, and brake B loosening. The frequency-variable motor accelerates the rotation at the set speed. (2) The backseat environment is loaded, the position sensor detects the flywheel position, and the IPC controls the clutch disconnection. Additionally, brake $\mathrm{A}$ is loosened, the impact hammer is released, and it collides with the specimen at a high speed. (3) Centrifugal loading is initiated, the IPC controls brake B latching, the impact hammer retracts, and the specimen rotates around the centrifugal track. (4) The simulation test results of $2 \mathrm{D}$ dynamic overloading are recorded in the acceleration storage test device.

In the process of fuze launching, the propellant burns rapidly, which produces immense bore pressure in the limited space. It then pushes the fuze to accelerate. In millisecond time, the axial velocity of the fuze rapidly changes from a static state to propelling hundreds of meters per second. In this motion state, the fuze achieves significant launch backseat acceleration. Because of the existence of the body pipe rifling, the fuze spins at a high speed. In the same millisecond time, its rotation speed changes from zero to thousands or even tens of thousands of revolutions per minute. In this overload environment, the fuze also attains considerable centrifugal acceleration. Figure 4(a) shows the $2 \mathrm{D}$ overload environment of the fuze during launching. The loading method described herein is used to simulate the launching backseat overload by means of the impact hammer striking the specimen at a high speed after suddenly accelerating from the stationary state. The function of the buffer is to obtain an acceleration-time curve that is close to reality through its own deformation. Buffers with various thicknesses and areas can be used to control the peak value and pulse width of the backseat acceleration. When the collision is completed, the specimen rotates in the track instead of spinning around its axis to simulate the centrifugal overload during launching. This method improves the peak value of the centrifugal acceleration by increasing the centrifugal radius. The simulation principle is shown in Figure 4(b).

\section{Dynamic Analysis of the Loading System}

3.1. Speed of the Flywheel and Backseat Acceleration. The backseat acceleration is produced by the impact hammer colliding with the test specimen at a high speed. The peak value and pulse width of the backseat acceleration are related to the flywheel speed. Therefore, it is necessary to analyze the collision process of the flywheel subsystem. Since the kinetic energy of the flywheel is significantly high and the collision contact time is considerably short (approximately $300 \mu \mathrm{s}$ ), it is assumed that the kinetic energy and momentum instant of the flywheel subsystem are conserved during the collision process. Therefore, the angular velocities of the flywheel before and after the collision can be written as

$$
\left\{\begin{array}{l}
\frac{1}{2} J \omega_{0}^{2}+\frac{1}{2} m_{\mathrm{sp}} v_{0}^{2}=\frac{1}{2} J \omega_{1}^{2}+\frac{1}{2} m_{\mathrm{sp}} v_{1}^{2} \\
J \omega_{0}+m_{\mathrm{sp}} v_{0} r_{\mathrm{sp}}=J \omega_{1}+m_{\mathrm{sp}} v_{1} r_{\mathrm{sp}}
\end{array}\right.
$$

where $J=22.63 \mathrm{~kg} \cdot \mathrm{m}^{2}$, which is the moment of inertia of the flywheel, $r_{\mathrm{sp}}=0.58 \mathrm{~m}$, which is the distance between the test specimen and the track center, and $m_{\mathrm{sp}}=0.78 \mathrm{~kg}$, which denotes the mass of the test specimen. Moreover, $\omega_{0}$ and $\omega_{1}$ are the angular velocities of the flywheel before and after the collision, respectively, and $v_{0}$ and $v_{1}$ represent the velocities of the test specimen before and after the collision.

According to equation (1), the angular velocity of the flywheel before the collision can be obtained by

$$
\omega_{0}=\frac{m_{\mathrm{sp}} v_{1} r_{\mathrm{sp}}^{2}+J v_{1}}{2 J r_{\mathrm{sp}}} .
$$

Assuming that the backseat acceleration is a half-sine waveform, the maximum velocity of the test specimen after the collision can be written as

$$
\left\{\begin{array}{l}
\bar{A}=\frac{\omega}{\pi} \int_{0}^{\pi / \omega} A_{b} \sin \omega t \mathrm{~d} t=\frac{2}{\pi} A_{b}, \\
v_{\max }=\int_{0}^{\tau} \bar{A} \mathrm{~d} t=\frac{2}{\pi} A_{b} \tau,
\end{array}\right.
$$

where $\bar{A}$ is the average backseat acceleration of the test specimen, $A_{b}=20,000 \mathrm{~g}$, which denotes the peak value of the backseat acceleration, and $\tau=300 \mu \mathrm{s}$, which is the pulse width of the backseat acceleration. The calculated $v_{\max }$ is $38.16 \mathrm{~m} / \mathrm{s}$.

Substituting $v_{\max }$ into equation (2), we have

$$
\omega_{0}=\frac{m_{\mathrm{sp}} v_{1} r_{\mathrm{sp}}^{2}+J v_{1}}{2 J r_{\mathrm{sp}}}=33.28 \mathrm{rad} / \mathrm{s} .
$$

Hence, the corresponding speed of the flywheel is $318 \mathrm{r} / \mathrm{min}$.

3.2. Speed of the Flywheel and Centrifugal Acceleration. The centrifugal acceleration relates to the velocity of the test specimen rotating around the centrifugal track. The centrifugal acceleration of the specimen can be written as

$$
A_{c}=\frac{v_{1}^{*^{2}}}{r_{\mathrm{sp}}}
$$

where $v_{1}^{*}$ is the velocity of the specimen after the collision and $A_{c}=1,000 \mathrm{~g}$, which represents the peak value of the centrifugal acceleration. The calculated $v_{1}^{*}$ is $75.42 \mathrm{~m} / \mathrm{s}$.

Substituting $v_{1}^{*}$ in equation (2), we have

$$
\omega_{0}^{*}=\frac{m_{\mathrm{sp}} v_{1}^{*} r_{\mathrm{sp}}^{2}+J v_{1}^{*}}{2 J r_{\mathrm{sp}}}=65.77 \mathrm{rad} / \mathrm{s}
$$

The corresponding motor speed of the flywheel is $628 \mathrm{r} / \mathrm{min}$. 


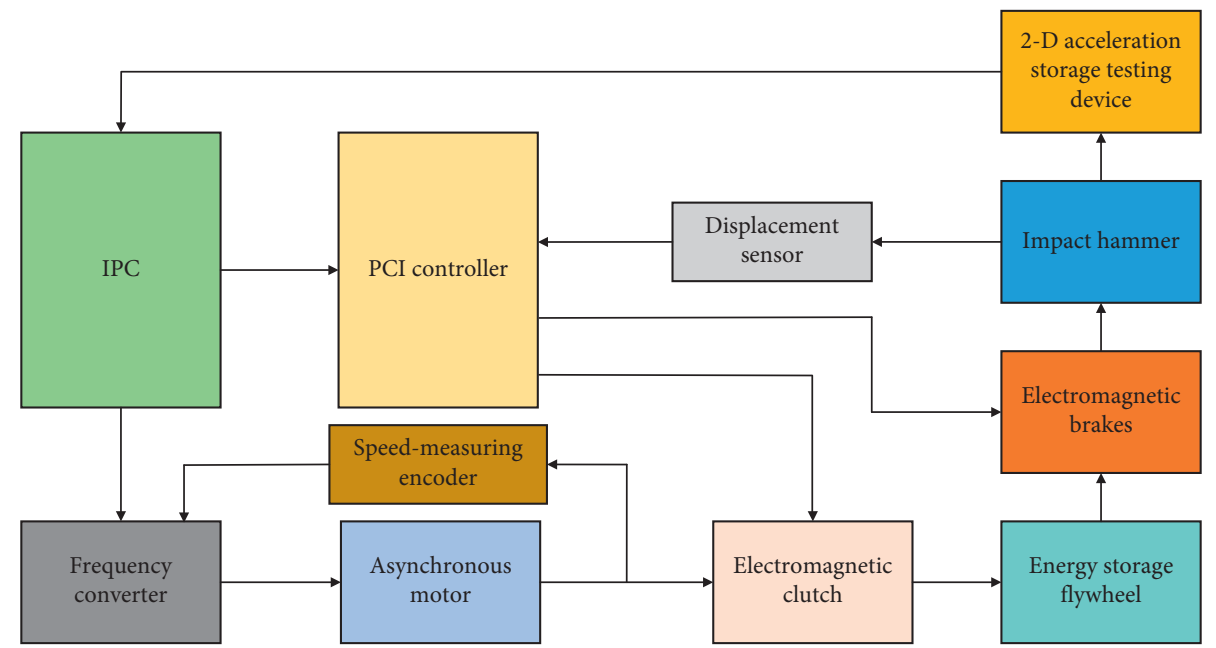

FIGURE 3: Control block diagram of the loading system of the $2 \mathrm{D}$ dynamic acceleration environment.

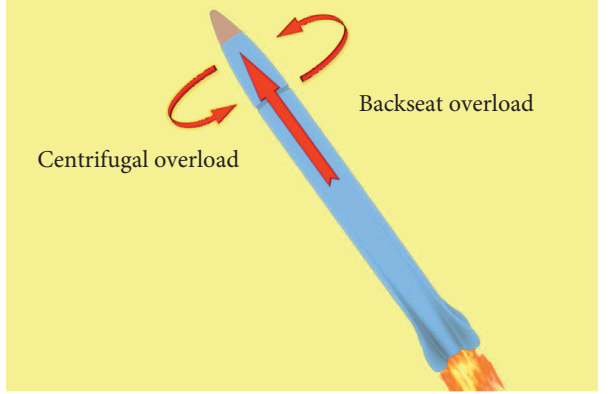

(a)

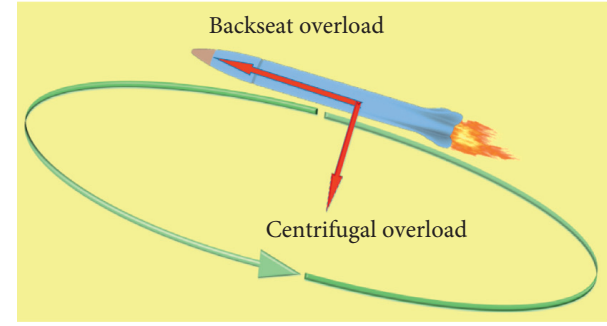

(b)

Figure 4: Simulation principle of the fuze 2D dynamic overload environment. (a) Actual overload environment of fuze launching. (b) Simulated overload environment of fuze launching.

In engineering practice, a good high-impact wide-pulse waveform is often obtained by adjusting the thickness and area of the buffers. The pulse width of the backseat acceleration can be obtained by [10]

$$
\left\{\begin{array}{l}
\tau=\pi \sqrt{\frac{\mathrm{mM}}{[K(m+M)]}}, \\
K=\frac{\mathrm{EA}}{L}
\end{array}\right.
$$

where $\tau$ is the pulse width of the backseat acceleration, $m$ denotes the test specimen mass with a buffer, and $M$ is the equivalent mass of the flywheel. Furthermore, $K$ denotes the system stiffness coefficient, $E$ is the modulus of elasticity of the buffer material, $A$ represents the buffer area, and $L$ is the buffer thickness.

Equation (7) shows that the duration of the backseat acceleration pulse width relates only to the material and structure of the buffers, not the collision velocity. Increasing the buffer thickness or reducing the buffer area can reduce the system stiffness and increase the pulse width duration.
The flywheel speed range is herein set at $600-900 \mathrm{r} / \mathrm{min}$ to simultaneously meet the requirements of both backseat and centrifugal accelerations.

\subsection{Calculation of the Brake Force and the Impact Hammer} Mechanism. To determine the force of the brakes, it is necessary to conduct a mechanical analysis of the impact hammer. A dimensional diagram of the brake force and the impact hammer mechanism is shown in Figure 5.

The centrifugal force $\left(F_{0}\right)$ on the impact hammer when the flywheel reaches the set value of $900 \mathrm{r} / \mathrm{min}$ is

$$
F_{0}=m_{h} \omega^{2} r_{0}=3198 \mathrm{~N} \text {, }
$$

where $m_{h}=1.5 \mathrm{~kg}$, which is the mass of the impact hammer, and $r_{0}=0.24 \mathrm{~m}$, which is the distance between the impact hammer centroid and the cylinder center.

The impact hammer is wound on the cylinder by a wire rope. The bending moment of the wire rope to the cylinder is

$$
M_{1}=F_{0} r_{c}=192 \mathrm{~N} \cdot \mathrm{m},
$$

where $r_{c}=0.06 \mathrm{~m}$ is the cylinder radius. The impact hammer should not extend until the flywheel reaches the set value. The force of brake A satisfies 


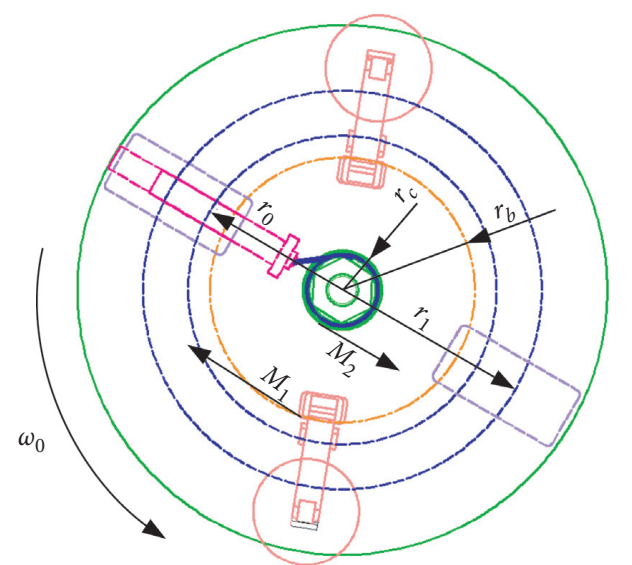

Figure 5: Dimensional diagram of the brake force and the impact hammer mechanism.

$$
F_{\mu A} \geq \frac{M_{1}}{r_{b}}=960 \mathrm{~N}
$$

where $r_{b}=0.2 \mathrm{~m}$ is the brake disc radius. The force of brake A is maintained at a constant $1200 \mathrm{~N}$ throughout this study.

The impact hammer is released and collides with the specimen. The backseat acceleration is loaded. The centrifugal force $\left(F_{1}\right)$ on the impact hammer can be written as

$$
F_{1}=m_{h} \omega^{2} r_{1}=4797 \mathrm{~N}
$$

where $r_{2}=0.36 \mathrm{~m}$ is the distance between the impact hammer centroid and the cylinder center. In the same way, the bending moment of the wire rope to the cylinder is $288 \mathrm{~N} \cdot \mathrm{m}$, and the force of brake B is $2000 \mathrm{~N}$.

3.4. Verification of the Spindle Strength. The strength and rigidity of the spindle must also be checked. The maximum torque of the spindle occurs while brake B is working. It can be obtained by

$$
M_{\max }=F_{\mu B} r_{b}=400 \mathrm{~N} \cdot \mathrm{m},
$$

where $F_{\mu B}=2000 \mathrm{~N}$ is the force of brake B.

The section modulus in the bending of the spindle center is

$$
W_{P}=\frac{I_{P}}{D / 2}=\frac{\pi D^{3}}{16}\left(1-\alpha^{4}\right)=3.98 \times 10^{-5} \mathrm{~m}^{3},
$$

where $D=0.06 \mathrm{~m}$ is the spindle diameter and $\alpha=0.5$ is the ratio of the inner diameter to the outer diameter of the spindle.

The maximum shear stress of the spindle center is

$$
\tau_{\max }=\frac{M_{\max }}{W_{P}}=10.06 \mathrm{MPa} .
$$

The maximum bending angle of the spindle center is

$$
\varphi_{\max }=\frac{M_{\max } L}{\mathrm{GI}_{P}} \times \frac{180}{\pi}=0.48\left({ }^{\circ}\right) / \mathrm{m} .
$$

The material of the spindle is $45 \#$ steel, and the safety factor is 1.5. Therefore, the allowable shear stress and twist angle are

$$
\left\{\begin{array}{l}
\tau=\frac{[\tau]}{1.5}=\frac{40}{1.5}=26.67 \mathrm{MPa} \\
\varphi=\frac{[\varphi]}{1.5}=\frac{1.5}{1.5}=1\left(^{\circ}\right) / \mathrm{m} .
\end{array}\right.
$$

The maximum shear stress and torsion angle are less than the abovementioned allowable value; therefore, the strength and rigidity of the spindle meet the given requirements.

\section{Speed Control Strategy of the Asynchronous Motor}

4.1. Design of the Fuzzy PID Controller. To improve the performance of the asynchronous motor, a speed regulator based on a fuzzy PID controller is presented in this paper. As observed in Figure 6, the regulator takes speed error $e$ and error rate $e c$ as input and modifies the PID parameters by using the fuzzy controller.

The fuzzy controller-modified PID parameters can be written as

$$
\left\{\begin{array}{l}
K_{p}(k)=K_{p}(k-1)+\Delta K_{p} \\
K_{i}(k)=K_{i}(k-1)+\Delta K_{i}, \\
K_{d}(k)=K_{d}(k-1)+\Delta K_{d}
\end{array}\right.
$$

where $K_{p}(k)$ is the adjusted proportional coefficient, $K_{i}(k)$ represents the adjusted integral coefficient, and $K_{d}(k)$ denotes the adjusted differential coefficient. Additionally, $K_{p}(k-1)$ is the proportional coefficient before adjustment, $K_{i}(k-1)$ represents the integral coefficient before adjustment, $K_{d}(k-1)$ denotes the differential coefficient before adjustment, and $\Delta K_{p}$ is the proportional coefficient of the fuzzy controller output. Furthermore, $\Delta K_{i}$ denotes the integral coefficient of the fuzzy controller output and $\Delta K_{d}$ is the differential coefficient of the fuzzy controller output.

Here, we define $e, e c$, and $\Delta K_{p}, \Delta K_{i}, \Delta K_{d}$ fuzzy subsets as \{NB (Negative Big), NM (Negative Middle), NS (Negative Small), ZO (Zero), PS (Positive Small), PM (Positive Middle), PB (Positive Big) $\}$ and their universe as $[-3,+3]$. The input and output fuzzy control rules are shown in Table 1.

4.2. Simulation of the Asynchronous Motor Speed Control System. To validate the asynchronous motor speed control system, Matlab/Simulink simulation analysis was conducted. The asynchronous motor parameters are shown in Table 2. The speed of the asynchronous motor was set at $800 \mathrm{r} / \mathrm{min}$, and the motor started with $50 \mathrm{Nm}$ torque as the load. The load suddenly disappeared at $3 \mathrm{~s}$.

The speed and torque simulation curves of the asynchronous motor are shown in Figure 7 . The motor speed requires $0.5 \mathrm{~s}$ for $0-800 \mathrm{r} / \mathrm{min}$ and enters a stable state without exceeding the set value. The load suddenly disappears at $3 \mathrm{~s}$, and the motor speed increases. The speed 


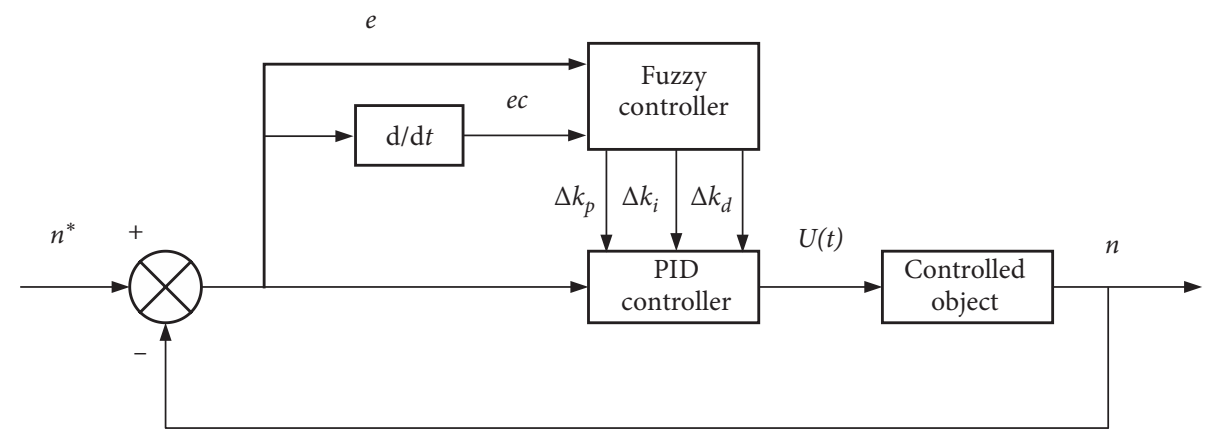

Figure 6: Principle diagram of the fuzzy PID controller.

TABLE 1: Fuzzy rules of $\Delta K_{p}, \Delta K_{i}$, and $\Delta K_{d}$.

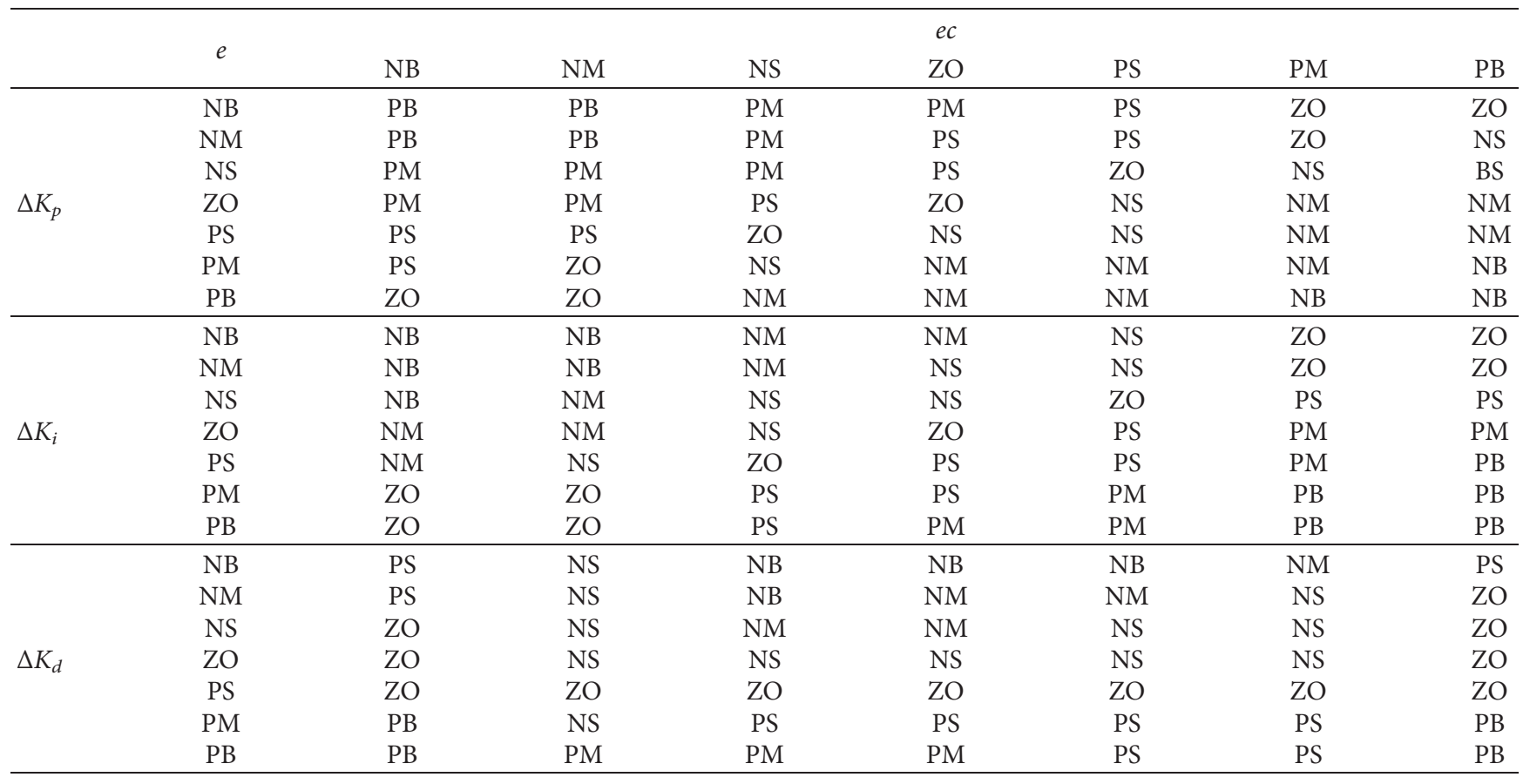

TABle 2: Parameters of the asynchronous motor.

\begin{tabular}{lc}
\hline Parameter & Value \\
\hline Polar logarithm $\left(p_{n}\right)$ & 2 \\
Rated voltage $\left(V_{n}\right)(\mathrm{V})$ & 380 \\
Stator resistance $\left(R_{s}\right)(\Omega)$ & 0.435 \\
Rotor resistance $\left(R_{r}\right)(\Omega)$ & 0.816 \\
Stator leakage inductance $\left(L_{\text {is }}\right)(\mathrm{H})$ & 0.002 \\
Rotor leakage inductance $\left(L_{\text {ir }}\right)(\mathrm{H})$ & 0.002 \\
Mutual inductance between stator and rotor $\left(L_{m}\right)(\mathrm{H})$ & 0.069 \\
Motor inertia $\left(J_{m}\right)\left(\mathrm{kg} \cdot \mathrm{m}^{2}\right)$ & 0.18 \\
\hline
\end{tabular}

can be quickly adjusted and returns to a steady state under the action of the fuzzy PID controller.

\section{Simulation and Test of the Loading Method}

5.1. Simulation of the 2D Dynamic Acceleration Environment. LS-DYNA software was used for the collision dynamics simulation. The simulation model is shown in Figure 8. The flywheel can be regarded as a block of mass that collides with the test specimen at a high speed. The equivalent mass of the flywheel is $90 \mathrm{~kg}$, the specimen mass is $0.78 \mathrm{~kg}$, and the buffer mass is $0.3 \mathrm{~kg}$. The material models and related parameters of each component are shown in Table 3.

Four sets of simulations were performed to verify the influence of the flywheel speed on the 2D dynamic acceleration, which ranged from 600 to $900 \mathrm{r} / \mathrm{min}$ with an interval of $100 \mathrm{r} / \mathrm{min}$. A buffer with a thickness of $30 \mathrm{~mm}$ and a diameter of $50 \mathrm{~mm}$ was employed in the impact simulation.

Figure 9 shows that the peak values of the backseat acceleration and centrifugal acceleration increase with the increase of the flywheel speed. All the backseat acceleration peak values are larger than the requirement $(20,000 \mathrm{~g})$, with a maximum of $48,264 \mathrm{~g}$. The pulse width of the backseat acceleration is approximately $310 \mu \mathrm{s}$, which likewise fulfills the requirement. The change of the flywheel speed has a minimal effect on the pulse width of the backseat acceleration, proving the accuracy of equation (7). Meanwhile, the centrifugal acceleration ranges from 611 to $1544 \mathrm{~g}$. 


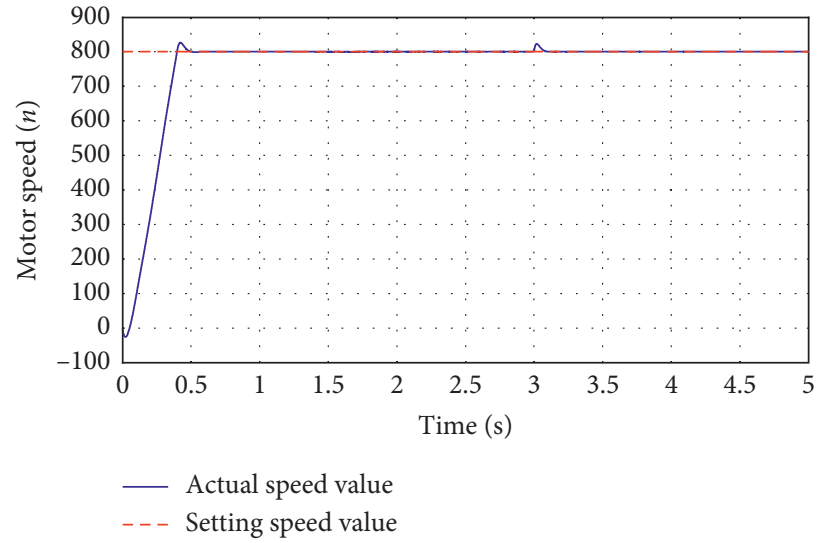

(a)

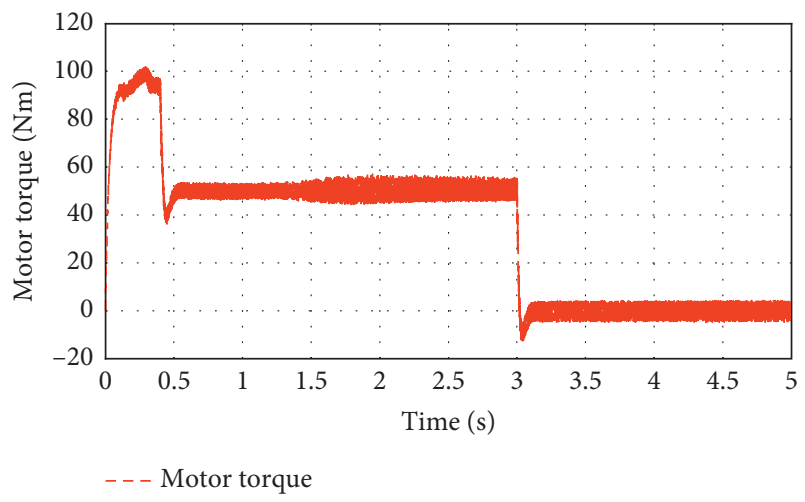

(b)

Figure 7: Simulation curve of the asynchronous motor. (a) Speed simulation curve. (b) Torque simulation.
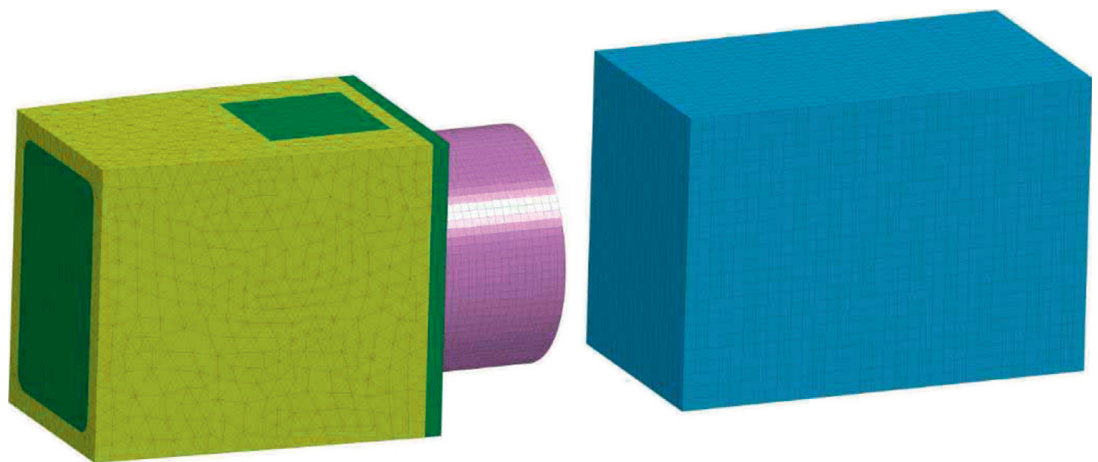

FIGURE 8: Simulation model of the impact hammer colliding with the test specimen.

TABLE 3: Material model and parameters of each component.

\begin{tabular}{lccc}
\hline & Mass block & Specimen & Buffer \\
\hline Materials & Steel & Aluminum & Rubber \\
Models & JOHNSON_COOK & JOHNSON_COOK & RIVLIN_RUBBER \\
Density $\left(\mathrm{g} \cdot \mathrm{m}^{-3}\right)$ & 7.83 & 2.77 & 0.94 \\
Young's modulus $(\mathrm{GPa})$ & 210 & 71 & 1.04 \\
Poisson's ratio & 0.28 & 0.33 & 0.42 \\
\hline
\end{tabular}

In this study, the pulse width of the backseat acceleration was increased by adding a buffer. We know that the pulse width of the backseat acceleration relates to the buffer thickness and area (equation (7)). First, four sets of simulations were conducted to verify the influence of the buffer thickness on the backseat acceleration. The speed of the flywheel was set as $800 \mathrm{r} / \mathrm{min}$, the buffer diameter was set to $50 \mathrm{~mm}$, and the buffer thickness was in the range of $20-50 \mathrm{~mm}$ with an interval of $10 \mathrm{~mm}$.

Figure 10 shows that, with the increase of the buffer thickness, the peak value of the backseat acceleration decreases, the rising time increases, and the pulse width prolongs. When the buffer thickness is $50 \mathrm{~mm}$, the peak value of the backseat acceleration decreases by $21 \%$ (approximately $37,977 \mathrm{~g}$ ) and the pulse width is approximately $382 \mu \mathrm{s}$, which meets the backseat overload requirement. The peak value of the centrifugal acceleration slightly increases with the buffer thickness increase.

Next, four additional sets of simulations were performed to verify the effect of the buffer area on the backseat acceleration. The flywheel speed was set as $800 \mathrm{r} / \mathrm{min}$, the buffer thickness was set to $50 \mathrm{~mm}$, and the buffer diameter range was $40-70 \mathrm{~mm}$ with an interval of $10 \mathrm{~mm}$. With the increase of the buffer area, the peak value of the backseat acceleration gradually increased, the rising edge became steeper, and the pulse width narrowed (Figure 11). The centrifugal acceleration did not significantly change.

From the simulation results, it can be observed that the peak value of the centrifugal acceleration, the peak value of the backseat acceleration, and the pulse width of the backseat 


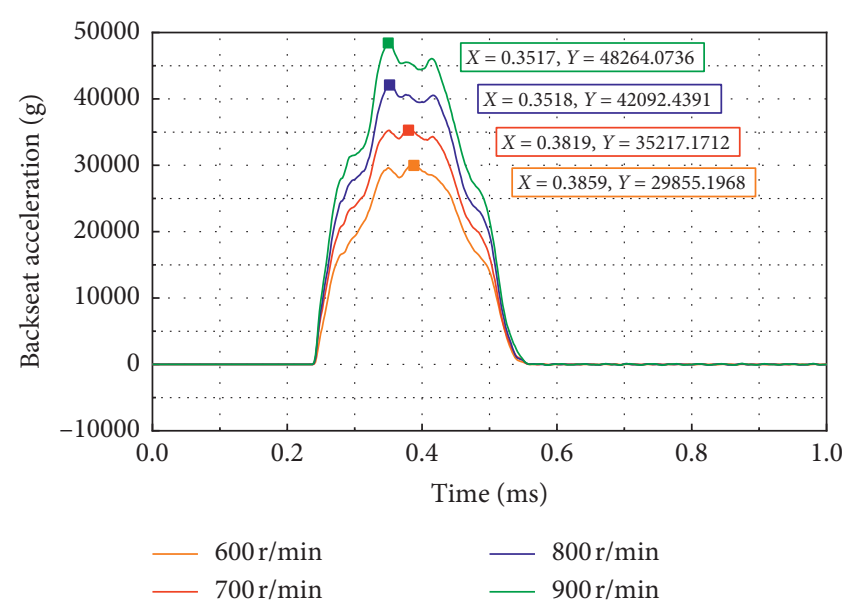

(a)

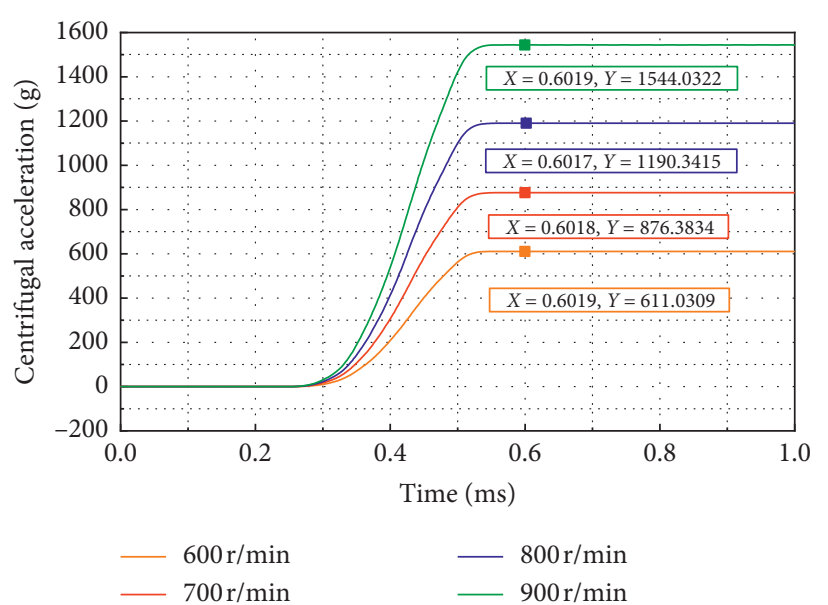

(b)

FIGURE 9: 2D acceleration simulation curves of the specimen at various flywheel speeds. (a) Backseat acceleration curve. (b) Centrifugal acceleration curve.

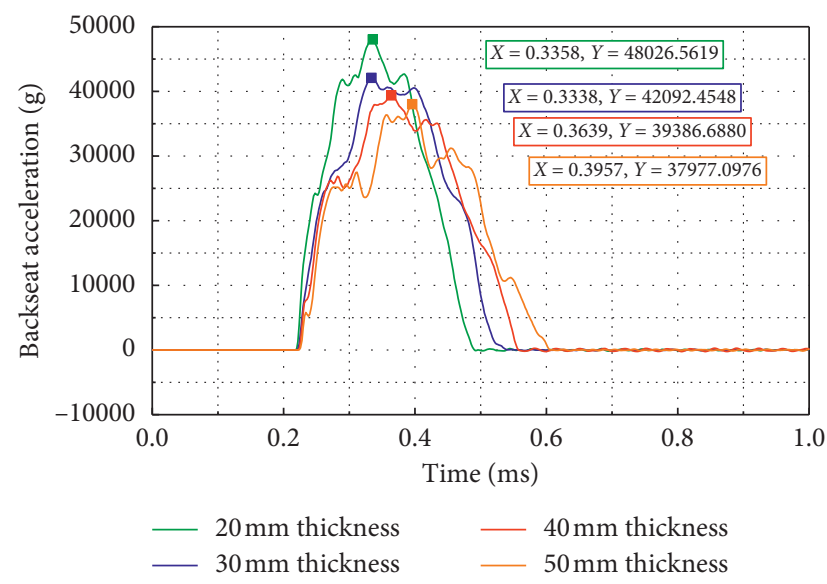

(a)

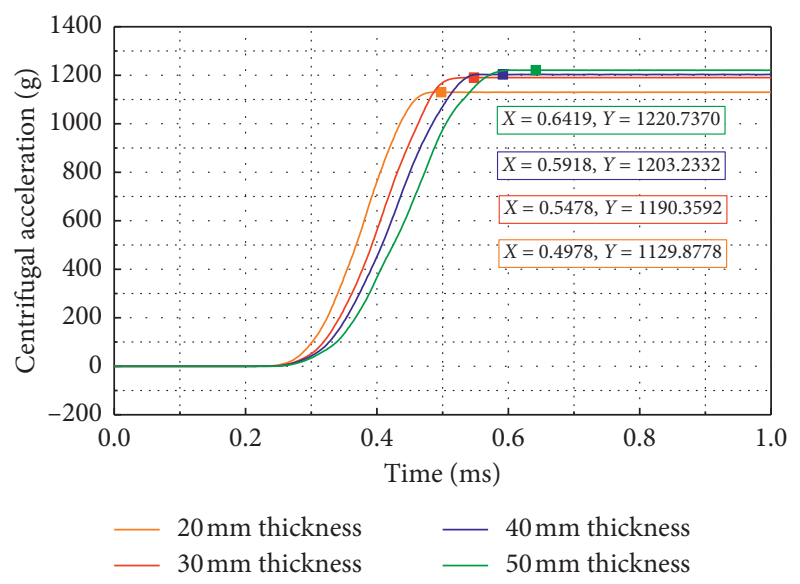

(b)

FIGURE 10: 2D acceleration simulation curves of the specimen with various buffer thicknesses. (a) Backseat acceleration curve. (b) Centrifugal acceleration curve.
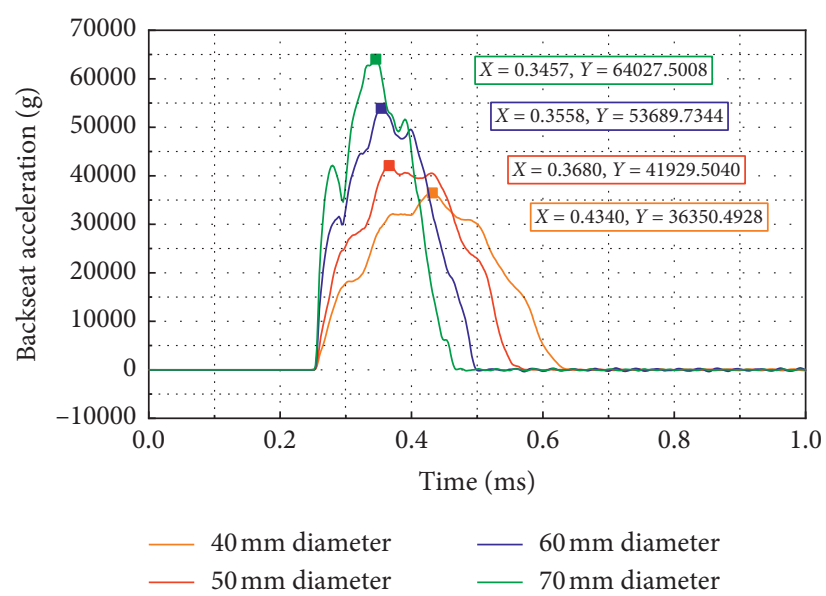

(a)

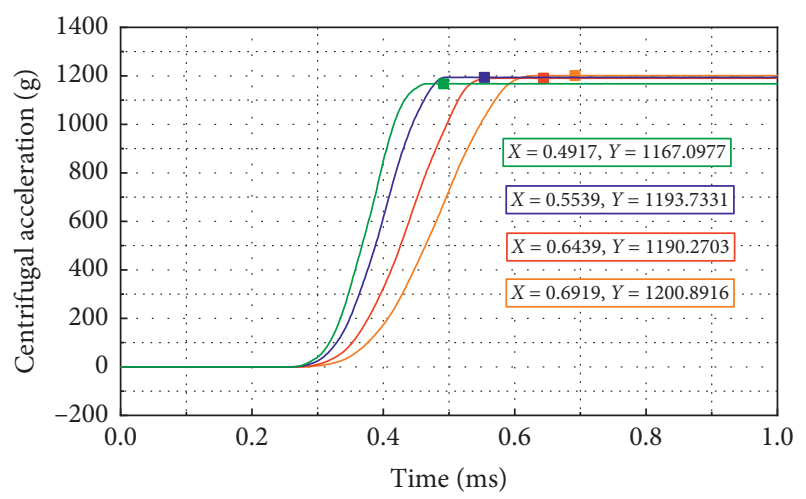

$\begin{array}{ll}-40 \mathrm{~mm} \text { diameter } & -60 \mathrm{~mm} \text { diameter } \\ -50 \mathrm{~mm} \text { diameter } & -70 \mathrm{~mm} \text { diameter }\end{array}$

(b)

Figure 11: 2D acceleration test curves of the specimen with various buffer diameters. (a) Backseat acceleration curve. (b) Centrifugal acceleration curve. 


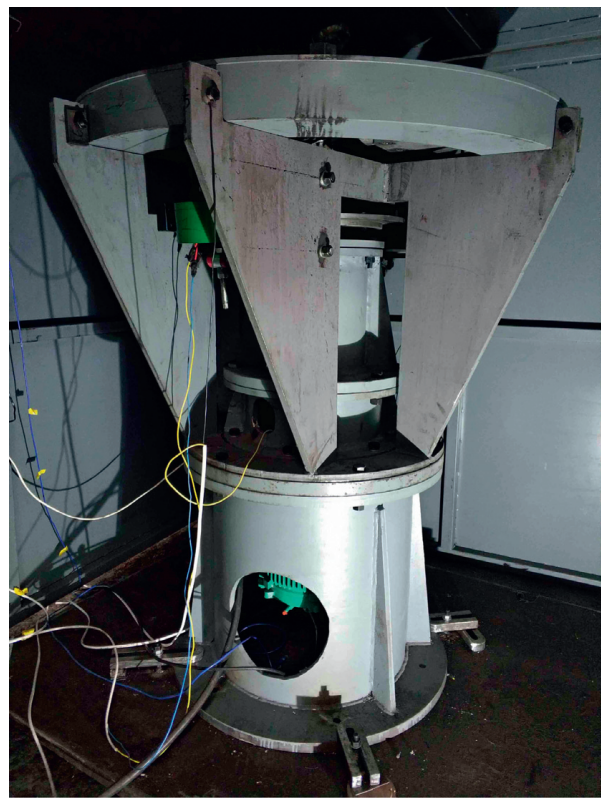

FIgURE 12: Photograph of the loading system of the 2D dynamic acceleration environment.

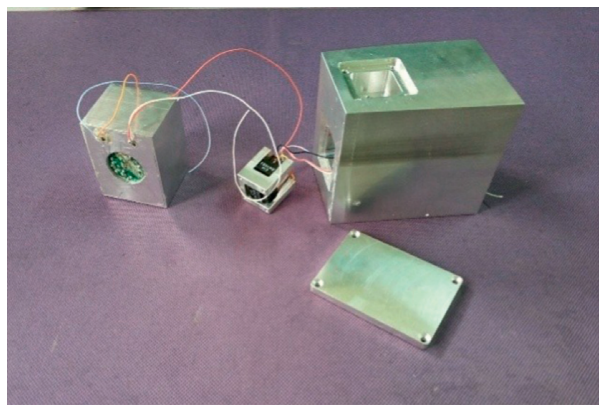

Figure 13: Photograph of the 2D acceleration storage test device.

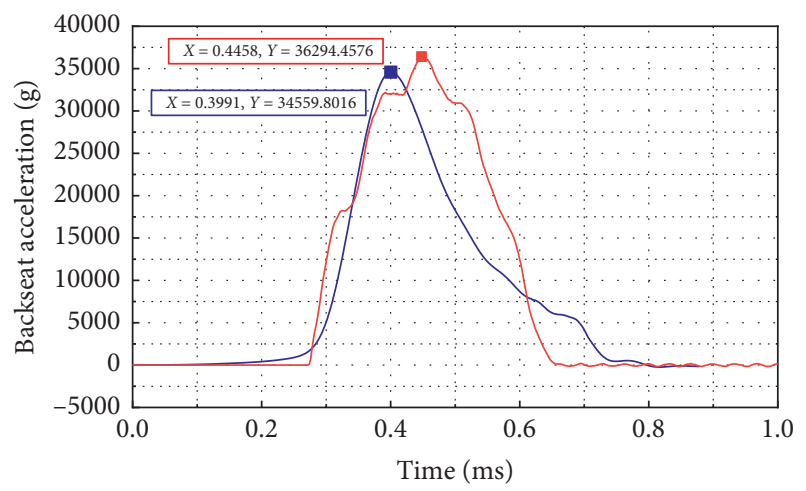

- Test backseat acceleration after filtering _ Simulated backseat acceleration

(a)

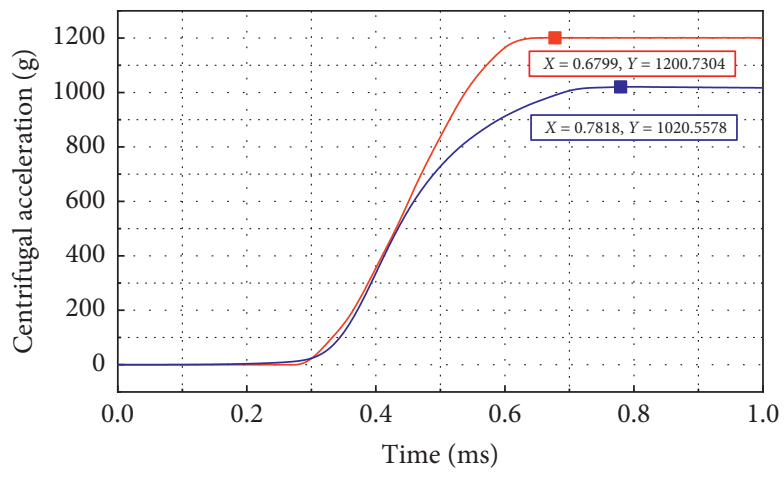

_ Test centrifugal acceleration after filtering _ Simulated centrifugal acceleration

(b)

Figure 14: Comparison of the filtered 2D acceleration curve obtained from the test and the simulation curve. (a) Backseat acceleration curve. (b) Centrifugal acceleration curve. 
acceleration can be adjusted by changing the flywheel speed as well as the buffer thickness and area to meet the simulation test requirements of various fuzes.

5.2. Experimental Verification. Currently, the proposed loading method is being successfully employed in ground simulation tests of the MEMS fuze safety and arming mechanism. A photograph of the loading system is shown in Figure 12. A 2D acceleration storage test device is used to record the test data. A photograph of the storage test device is shown in Figure 13. The range of the backseat accelerometer is $80,000 \mathrm{~g}$ and that of the centrifugal accelerometer is $20,000 \mathrm{~g}$. The sampling frequency is $500 \mathrm{kHz} / \mathrm{channel}$.

In the simulation test conducted in this study, the speed of the energy storage flywheel was set at $800 \mathrm{r} / \mathrm{min}$, the acceleration time was $100 \mathrm{~s}$, the buffer diameter was $40 \mathrm{~mm}$, and the thickness was $50 \mathrm{~mm}$. A comparison of the filtered $2 \mathrm{D}$ acceleration curve obtained from the test and the simulation curve is shown in Figure 14. The peak value of the backseat acceleration reaches $34,559 \mathrm{~g}$, which is $1,735 \mathrm{~g}$ lower than the simulation curve, with a decrease of $4.78 \%$. The pulse width of the backseat acceleration can reach $400 \mu \mathrm{s}$, which is $34.03 \%$ longer than the simulation curve. The peak value of the centrifugal acceleration is $1,020 \mathrm{~g}$, which is $180 \mathrm{~g}$ lower than the simulation curve, with a decrease of $15.01 \%$. The simulation test results show that the $2 \mathrm{D}$ dynamic overload provided by the loading system meets the requirements of ground simulation test of the MEMS fuze safety and arming mechanism.

\section{Conclusions}

The fuze launch process is subjected to backseat and spin overloads. This paper presented a loading method of $2 \mathrm{D}$ dynamic acceleration environments to test on the ground the antioverload performance of the fuze. The techniques of flywheel energy storage, high-speed impact, and centrifugal rotation in the track are combined based on dynamic analysis and a simulation. A fuzzy PID strategy is used to control the motor speed. The structure and working principle of the loading system were herein detailed. The simulation and test results showed that the proposed method is satisfactory and effective. The main innovations and conclusions of this study are as follows:

(1) Use of an energy storage flywheel as the main hardware of the loading system can provide more energy than an air gun loading system. Moreover, this approach is more suitable for simulating the $2 \mathrm{D}$ dynamic overload environment with high " $G$ " acceleration. At the same time, the energy storage flywheel has the advantages of a simple structure, small floor area, and low cost.

(2) To simulate the centrifugal overload, a high-speed collision simulates the backseat overload when the fuze is launched, and a test specimen is used to rotate around the centrifugal track instead of spinning around its axis. By increasing the centrifugal radius, the flywheel speed can be effectively reduced, and the synchronous loading of the 2D dynamic overload environment can be easily realized.

(3) In the simulation test process, the peak value and pulse width of the $2 \mathrm{D}$ acceleration can be adjusted by changing the speed of the flywheel and different buffers.

(4) The experimental and simulation results showed that the peak value of the backseat acceleration could reach $34,559 \mathrm{~g}$, the pulse width was approximately $400 \mu \mathrm{s}$, and the peak value of the centrifugal acceleration was $1,020 \mathrm{~g}$. The requirements of the $2 \mathrm{D}$ overload simulation test of the MEMS fuze safety and arming mechanism were thus fulfilled.

(5) The errors of the peak value and pulse width of the backseat acceleration were $4.78 \%$ and $34.03 \%$, respectively, and that of the centrifugal acceleration provided by the loading system was $15.01 \%$. Pulse width extension is beneficial to the simulation test of the MEMS fuze safety and arming mechanism; thus, the loading system error can be considered $15.01 \%$.

(6) The proposed loading system has been successfully applied to ground simulation tests of the MEMS fuze safety and arming mechanism.

\section{Data Availability}

The data used to support the findings of this study are included within the article and can be used for other research studies.

\section{Conflicts of Interest}

The authors declare that there are no conflicts of interest regarding the publication of this paper.

\section{Acknowledgments}

We thank Editage (http://www.editage.cn) for its linguistic assistance during the preparation of this manuscript. This work was supported by the Key Laboratory Research Fund (Grant no. C120401).

\section{References}

[1] W. Wang, S. Bi, H. Xiang, C. Zhan, and X. Yuan, "Trigger control characteristics of fuze-recoil simulation system based on electromagnetic launcher," Journal of Systems Engineering and Electronics, vol. 28, no. 3, pp. 563-571, 2017.

[2] J. E. Field, S. M. Walley, W. G. Proud, H. T. Goldrein, and C. R. Siviour, "Review of experimental techniques for high rate deformation and shock studies," International Journal of Impact Engineering, vol. 30, no. 7, pp. 725-775, 2004.

[3] Y. Liu and B. Liaw, "Drop-weight impact tests and finite element modeling of cast acrylic/aluminum plates," Polymer Testing, vol. 28, no. 8, pp. 808-823, 2009.

[4] E. V. González, P. Maimí, P. P. Camanho, A. Turon, and J. A. Mayugo, "Simulation of drop-weight impact and compression after impact tests on composite laminates," Composite Structures, vol. 94, no. 11, pp. 3364-3378, 2012. 
[5] B. Yang, L. Tang, Y. Liu, Z. Liu, Z. Jiang, and D. Fang, "Localized deformation in aluminium foam during middle speed Hopkinson bar impact tests," Materials Science and Engineering: A, vol. 560, pp. 734-743, 2013.

[6] K. G. Rakvåg, T. Børvik, and O. S. Hopperstad, "A numerical study on the deformation and fracture modes of steel projectiles during Taylor bar impact tests," International Journal of Solids and Structures, vol. 51, no. 3-4, pp. 808-821, 2014.

[7] J. Xu, H. Chen, C. Tan, H. Chen, and G. Zhou, "Numerical and experimental investigations for an air cannon optimization," Science China Technological Sciences, vol. 54, no. 2, pp. 345351, 2011.

[8] W. Xia, S. Fan, W. Xing, T. Ma, J. Zu, and J. Fan, "Restraining zero drift in an ultrahigh-gimpact environment," Measurement Science and Technology, vol. 23, no. 3, Article ID 035108, 2012.

[9] C. Zhan, H. Xiang, and B. Lei, "Research on fuze dynamic simulation based on electromagnetic coil launcher," Journal of Academy of Armored Force Engineering, vol. 29, no. 2, pp. 56-60, 2015.

[10] W. Wang, S. Bi, and H. Xiang, "Electromagnetic shielding of overload experiment device for fuze based on electromagnetic driving technology," Acta Armamentarii, vol. 37, no. 5, pp. 909-915, 2016.

[11] Y. Zhang, T. Ma, J. Zu, and J. Sun, "Vector turntable centrifuge started by air cannon for missile launching simulation," Journal of Detection \& Control, vol. 34, no. 1, pp. 28-32, 2012.

[12] H. Xiang, B. Lei, and L. Chen, "Simulation system of two environmental forces for fuse based on single chip computer," Armament Automation, vol. 24, no. 6, pp. 40-41, 2005.

[13] P. Wang, L. Sui, and G. Li, "A MEMS safe and arming system load simulation device," Journal of Detection \& Control, vol. 37, no. 6, pp. 17-20, 2015.

[14] Y. Zhang, T. Ma, and J. Sun, "Simulation methods of twodimensional acceleration overload environment for missiles based on turntable and centrifuge," Journal of Projectiles, Rockets, Missiles and Guidance, vol. 36, no. 4, pp. 109-112, 2016.

[15] X. Qi, Q. Yang, J. Wen, and L. Cui, "Summary of fuze dynamic simulation based on gas gun," Journal of Detection \& Control, vol. 33, no. 4, pp. 1-5, 2011.

[16] Q. Yang, X. Qi, Z. Zhao, Y. Li, and M. Han, "The influencing factors of recoil acceleration of fuze simulated by impact and deceleration method," Journal of Projectiles, Rockets, Missiles and Guidance, vol. 32, no. 3, pp. 111-113, 2012.

[17] H. Qian, R. Gao, and H. Wang, "Study on simulation of double environmental forces of liquid reserve battery for fuses," Structure \& Environment Engineering, vol. 40, no. 1, pp. 58-63, 2013.

[18] M. Xu, N. Yu, and J. Liu, "The dual-environment testing method of accumulator in fuze," Environmental Engineering, vol. 31, no. S1, pp. 729-732, 2013.

[19] C. Lin, "Study of setback and spin environment simulation of fuze in launch process base on electromagnetic emission technology," Master's thesis, North University of China, Taiyuan, China, 2017. 